\title{
Teaching and Learning in a Multi-Disciplinary World: Examples from a Sino- British University in China
}

\author{
Raffaele Pernice, Gareth Morris, Chengcheng Li, Ann Brantingham \\ $X i^{\prime}$ an Jiaotong-Liverpool University \\ China P.R.
}

\begin{abstract}
Multi-disciplinary teaching and learning is an emerging innovative educational form which harbours significant promise in terms of improving students' study experiences and practitioner's pedagogic practice in an era of higher education massification typified in many places by increased numbers of $L 2$ subject language learners. Drawing on some examples from four Joint Delivery modules which are currently offered at Xi'an Jiao Tong-Liverpool University (XJTLU) this paper provides a reflective and thought provoking discussion on the form, format and pedagogy currently underpinning some of the multidisciplinary teaching and learning experiences offered at this internationally emerging institution and proposes suggestions for good practice in terms of taking them forward in future years. These five credit modules offered in semester two of undergraduate students first year at Xi'an Jiao Tong-Liverpool University are supported by university' staff from the School of Film and Television Arts, the departments of English, Culture and Communication (ECC) and Urban Planning and Design (UPD), as well as the Language Centre (LC). Now into their fifth year, the modules have also undergone some reasonably substantial developments in terms of the syllabus, delivery pattern, content, learning space and staffing. In addition, ICT has also played a much greater role with the advancement of time. This paper will therefore highlight some of the evolutionary changes designed to enhance student learning whilst also illustrating how the courses are increasingly innovative.
\end{abstract}

\section{Introduction}

Multi-disciplinary teaching and learning is an emerging innovative educational form which harbours significant promise in terms of improving students' study experiences and practitioner's pedagogic practice in an era of higher education massification typified in many places by increased numbers of L2 subject language learners. Drawing on examples from two Joint Delivery modules which are currently offered at Xi'an JiaoTong Liverpool University (XJTLU) this paper provides a reflective and thought provoking discussion on the form, format and pedagogy currently underpinning some of the multidisciplinary teaching and learning experiences offered at this internationally emerging institution and proposes suggestions for good practice in terms of taking them forward in future years.

Xi'an Jiaotong Liverpool University (XJTLU) was established in 2006 as a Sino-British joint venture university between the University of Liverpool in the UK and Xi'an Jiaotong University in China. The two main campuses of this academic institution are both located in Dushu Lake Higher Education Town, Suzhou Industrial Park in the historic city of Suzhou, approximately $80 \mathrm{Km}$ west of Shanghai, China. All of the instruction at XJTLU is in English, but nearly all of the students are non-native English speakers. Students at the end of year 2 of the program of their choice have the option to move to the University of Liverpool to complete their degree, which is recognized by both the UK and Chinese ministries of education. XJTLU's vision is to become a research-led international university in China and a university recognised internationally for its unique features in learning and teaching, research, social service, and education managements. Great emphasis is put on the efforts to develop a new, symbiotic relationship with business, industry and the local community while continuously promoting high-calibre academic research. Xi'an Jiaotong Liverpool University has an international body of faculty from both the East and the West, and students, whose majority fundamentally are from mainland China with a minority (less than $10 \%$ ) from outside of China, focus on innovation and internationalization, therefore approximately $80 \%$ of graduates would choose to go abroad to further pursue postgraduate studies. 


\section{Background}

Incorporating multi-disciplinary teaching methods by embedding language instruction within an academic content module and combining language teachers and academic instructors in the same classroom was seen by XJTLU as a viable approach to improve the learning and teaching experience of the students, particularly in year 1 . Thus, the Joint Delivery modules at XJTLU originated out of concern over students struggling with the synthesis of language and academic content and to act as a bridge to assist in the transition from year 1 which has a language and study skills focus to years 2-4 which are subject specific focused. Because the first year of study at XJTLU is quite heavily weighted on language and study skills centered on 10 hours per week of EAP (English for Academic Purposes), there was a need to help students carry over those academic English skills into their subject specific modules. The Joint Delivery modules are a way to embed the English language and study skills instruction into their academic study. They also facilitate greater communication between the Language Centre and the academic departments regarding integrating language and study skills development throughout their university careers [8].In terms of students' English levels, those Joint Delivery Modules are designed for year $1 / 2 / 3$ students who use English a second language and who are studying academic courses in English. The majority of them have little experience interacting with native speakers of English and little inter-culture communication experience. They have difficulties understanding not only the meaning of academic concepts, instructions of faculties, but also difficulties in constructing knowledge in English, making connections, as well as communicating ideas in appropriate contexts and academic styles. The language barrier also poses difficulties in student motivation which may have profound but immeasurable effect in their learning experience which makes the Joint Delivery necessary and beneficial in both obvious and discrete ways. The first module was delivered in 2013 with the collaboration of three departments (the Language Centre, the Department of English, Culture and Communication, and the Department of Urban Planning and Design). That original "pilot" module proved successful and has grown into the "Joint Delivery" sub-department of the Language Centre. Currently (2017/18 academic year), there are 30 Joint or Assisted Delivery modules planned and delivered by 13 collaborating departments and the Language Centre. There are a variety of module formats (either "Joint Delivery" or "Assisted Delivery") available for collaboration between the Language Centre and the academic departments. The chosen format will determine which department manages the different aspects of the module. This includes module ownership, syllabus content, materials development, teaching, contact hours, assessment development and assessment marking.

\section{Consideration 1: Syllabus Design}

For those tasked with teaching on any Joint Delivery module or course one of the first considerations is, somewhat obviously, syllabus design. This involves many potential features as Brown and Richards note, such as ideally conducting a needs analysis, reviewing or setting goals and objectives, considering the structure, content and methodology of the offering, designing or adopting materials for in or after class use, as well as creating assessments which are fit for purpose [4] [11]. There should also be an evaluation or reflection process both during and after the course or module. In the case of many Joint Delivery modules this necessitates, firstly reflecting on the suitability and teachability of the module learning outcomes, which may well cover a diverse range of specialist expectations. It also means taking into account learner backgrounds, with many students potentially learning subject specialist content that is very new to them based on their previous study experiences, which is not to mention the obvious L2 language challenges many students face. For the module convener there is the additional challenge of trying to sync a syllabus that necessitates staff provision from a number of different departments meaning that unavailability for certain weeks can create rethinks in terms of sequencing and a compromise on coherence potentially. With materials also coming from different specialisms and individuals collating all of this, and providing language support sessions to complement specialist input, additional difficulties can arise if delays or absences occur. Planning for assessments also necessitates careful forethought as assessing the learning outcomes is obviously essential, but assessment familiarity should also not be overlooked. If it is, or issues arise anywhere in the process already mentioned then it is likely that student feedback at the courses end and staff feedback via observations, or assessment statistics will highlight potential issues. With regards to personal experiences it is believed that looking forward, learning outcomes should have both clarity and assessability, and bear broader programme relevance. The needs analysis should encompass consideration of the learner's language starting points and subject specialist knowledge, staff capabilities and departmental aims. The structure and content needs to additionally be mindful of departmental staffing 
constraints and personal changes, but not to the extent that course coherence is compromised. Additionally, materials should be archived (as they usually are) and provide a resource bank for the future, with assessments designed to ensure learning outcomes are being assessed, marking loads are manageable, and any descriptors easily understood and usable. Ultimately, at the end of the course the hope is that evolutionary fine tuning and not major overhauls are the norm.

For one Joint Delivery module titled 'Imagining the City' many of the above considerations came to the fore. Although a formal needs analysis was not conducted due to time constraints, and the fact that the syllabus has to be specified to some extent in the module specifications prior to its commencement, many of the other constituent features of syllabus design featured prominently during the course of the semester. The exact topics of the curriculum were discussed and agreed in advance, the delivery order of these was carefully considered to take into account assessment sequencing and course coherence; materials were devised in light of target weekly learning outcomes as well as longer term course understanding and assessment needs. Assessments also went through a very rigorous process of moderation, and the marking likewise. There was also a thorough reflection on the course at the end based on module questionnaire feedback from students, practitioner input and assessment data.

However, there were also a number of challenges which were encountered. Firstly, with regards to the ordering of the topics, there was a preference by some for an eclectic sequencing, albeit while still ensuring that the assessment sequence would be adhered to. Other voices preferred a more systematic block structure. Reality in this instance leant more towards the latter, but with a bit of flexibility inbuilt due to lecturer availability, as well as to account for unforeseen absences as and when they infrequently occurred. Fortunately, the results from the student feedback questionnaire, in which all learners have an opportunity to provide feedback on the course have been extremely positive all things considered. The feedback results have also reflected year on year improvements for the past three years. For example, responses to the final question querying the perceived value of the learning experience saw the numbers on a five point Likert scale progress from 3.95 in 2014-15 to 4.15 a year later, and 4.22 in $2016-17$. It is likely that module stability, in terms of staffing, and incremental improvements are paying dividends.

Another Joint Delivery module that is also benefiting from staff stability has been MAN001 or 'Business Essentials' as it is termed. This module is a business school year one module that is run with
Language Centre support. It has also moved from being mandatory to optional, but still has over 1200 enrolled students for the 2017-2018 academic year. The overall perceived student satisfaction with this module's value has also been improving with each academic year. In 2014-15 the mean average was 3.96; a year later it was 4.13 and this academic year just gone (2016-17) the figure was 4.38. These numbers are approaching regular EAP class averages, where the average class size is 20 and students have approximately 10 contact hours a week with their teacher. In contrast, the average seminar size for the aforementioned Joint Delivery modules is between 100 and 150, with lectures having hundreds of students. The reason behind the improvements is fairly obviously multifaceted, but a significant part results from sound planning. Ensuring that the basics of the syllabus are well designed, both procedurally and operationally, and in place in good time goes a long way to ensuring a greater chance of success. Acting on feedback and subsequently enacting incremental improvements also helps in this respect. Although there is still work to be done and areas in which improvements could still be made with regards to both modules, and of courses practical limitations to be factored in, the results to date have been extremely positive and the outlook remains so.

\section{Consideration 2: Individual Differences}

Another area which is obviously very important to consider when delivering a course relates to individual differences. These may be between staff on a module that is joint delivered or co-taught, as well as between staff and students and students themselves. Clearly knowledge bases, expectations for teaching and learning, indeed almost every imaginable aspect related to the course may be perceived in very different ways by different people and groups of people. Focusing on the differences between students in the class and the individual differences which exist, as well as what the teacher can subsequently do to better facilitate learning, is incredibly important. For example, when students enroll on any module the knowledge they bring with them at the start of the course can be very different. Their learning styles and rationale and motivation for learning can also differ quite considerably. Language differences can also play a part as in an international classroom the language of delivery can either hinder or help students potentially. Drawing more specifically on the example of another Joint Delivery module titled 'Critical Thinking: The Self and the World' a number of these features and concerns resonated.

On this module students' existing knowledge, cooperating with different departments for content 
input, helping learners construct meaning from content, as well as applying problem-posing pedagogy were valued, as they are in most Joint Delivery teaching practice. To begin with, and with the aforementioned module in mind, through exploration of funds of knowledge [10], teachers could help to establish symmetrical relationship with students in that students' knowledge can be developed into classroom teaching resources, which encourages learners to form connections with course ideas and become more responsible learners. The nature of this module requires individual perspectives in that thinking is both a public and private process. For example, in learning "What is Truth", students were introduced to various ideas concerning the definition of truth. Yet, comprehension and understanding of the printed ideas are not adequate for thinking and defining truth because the thinking and defining process entails a very intimate and close investigation of personal life. Thus, much of the class is also devoted to help students be aware of their own life experience, thinking process, and how others' perspectives are different. Similarly, students partially construct their own identities and understanding of the world [9] before coming to the classroom, and also then within it. Their particular life experience helped form their particular beliefs which are taken for granted if not confronted with contradicting ones. And to be a critical thinker, one must develop skills that help oneself to look beyond the boundaries of personal beliefs. It is important therefore to challenge learners by eliciting ideas from their life experiences and inviting them to reflect, question, and challenge others' and their own beliefs.

As to assessing the learning outcomes, students' knowledge provides a good basis from which to build when setting goals and designs for measuring progress [1], [2] in that learners' existing knowledge can help teachers identify the gap between what is already known and what ought to be by the courses end. However, integrating individual differences in assessing learning outcomes also pose difficulties for the standard criteria is used to measure a group of diverse learners. How to best balance the application of assessment criteria and individual differences should be taken into consideration. One way to achieve that goal is to allow learner autonomy by providing open-ended questions, reflective essay tasks, presentation, and group projects. In this module, a reflective essay, group video project, and essay are used for assessing learning outcomes. The combination of different kinds of assessment allows learners to demonstrate their learning more effectively in that their individual differences are well integrated into the completion of assessment tasks.

In terms of working with abstract content provided by different departments, one approach used is to help learners construct meaning through actively engaging with content. Freire and Macedo mention that being able to reflect on one's experience is important for understanding the world and the limits and possibilities of the society which is crucial for developing critical thinking [6], and this is especially important in the Joint Delivery Module (LAN102), titled 'Critical Thinking: The Self and the Word.' Here students are instructed to construct meaning through reflecting on their own being and foster senses of introspection. With the assistance of the teacher, students may find texts which appear irrelevant and boring in fact telling and stimulating. In addition, the modes of learning draw ideas from critical pedagogy [5] based on which the goal of education is to help learners achieve awareness through challenging the status quo and understanding the relationship between oneself and the world including the student-teacher relationship [7]. Therefore, the teacher is viewed as the facilitator rather than the narrator of knowledge who imparts sage like wisdom to the empty minds of learners. In this vein of thinking, naturally, discussion becomes a main way for learners to take ownership of their class [3]. Furthermore, the modes of learning and teaching also view learners as diverse individuals who are from different cultures. Thus, teachers ought to be patient and tolerant of uncertainty in learner performance, especially as it is more effective to learn if students are more aware of their own culture frame rather than less aware [1].

A key way to provide language assistance in cooperation with many academic disciplines is through working with concepts and vocabulary. While language tutors are not expert in a specific academic discipline module, they can help with understanding concepts and vocabulary by putting them in the right contexts. These concepts and vocabulary are building blocks for knowledge of a specific field and it is the language tutors' role to help students understand the concepts and prompt students' thinking which entails the use of such concepts and vocabulary. Additionally, by helping students with studying skills, language tutors could help with cognition development that help learners with memorizing skills, evaluating skills, analysis skills which can be applied generally in understanding, synthesizing, and communicating ideas in a discipline using relevant concepts and vocabulary.

In addition, it is also worth mentioning that the individual difference between the teachers who taught this module. The different teaching styles and content of each teacher requires students' close cooperation. For example, in this module, students work with 4 different lecturers who are in charge of either one or two lectures. In addition, they work with one seminar tutor who would deliver 20 seminars. The individual 
difference and different teaching styles entails cooperation among the lecturers to ensure the cohesion of content. This also compels Joint Delivery instructors to be sensitive and conscious of maintaining coherence in students' expectation of degrees of regularity in teaching styles and make students more alert and informed thus prepared to cooperate with teachers of different styles. In order to ensure quality delivery of content, the seminar tutor is required to sit through each lecture to take notes in addition to pre and post-lecture discussions. The Joint Delivery instructors are also required to help students understand the connection between different lecturers' ideas which are not explicitly connected given the nature of this module. Students would be informed of an overview of the content before the lecture in announcements in which ideas from previous lecture and the current lecture are linked logically and cohesively.

After two cohorts of the Joint Delivery Module (LAN 102), the module leader came to realize that the implementing of individual difference in designing class activities has very positive results. For example, students expressed in their questionnaire that this module has deeply affected their thinking and life. Others' commented on how their personal views are respected and valued through the discussion-featured ways of teaching and learning. Again, many also expressed that they learned many different perspectives by expressing themselves and listening to peers. One thing worth mentioning in the discussion of individual difference is how teachers play an important role in helping students find appropriate group members and helping facilitate their communication among peers.

\section{Consideration 3: Teaching and Learning Space}

In an attempt to foster discussion in the classroom between students and between the students and instructor, the teaching space should be considered as a significant aspect of multidisciplinary teaching and learning. The teaching space is as influential on learning as the teaching method and the space often dictates the method [12]. This was a key issue to be addressed based on feedback from the first Joint Delivery module's completion in 2013. Traditionally, large classes are assigned to the lecture theatre with the assumption that lecture style teaching is the only or best method for such situations. In addition to that, at XJTLU, there were no large flat classrooms available for regular weekly classes; therefore, the first Joint Delivery module with 175 students was delivered in the lecture theatre. Even so, the combined teaching staff of Language Centre tutors and academic staff struggled to deliver communicative style teaching in a tiered classroom with fixed seating. The feedback about the teaching space was well received by management and the multidisciplinary methods of Joint Delivery modules at XJTLU fostered an adjustment to the teaching space in an attempt towards innovation to the teaching delivery space and method. Moving lectures from the traditional tiered lecture theatres to a flat flexible learning space was deemed a necessary change in order to have a positive influence on the students' learning experience. Thus, new spaces were constructed which included movable round tables (8 seats) in a room with a capacity for 130 students. By delivering lectures to large classes (80120 students) in a large flat space with movable tables and chairs, the lecturer is able to be more interactive with the students, and it promotes student interaction which has been shown to stimulate learning [12]. The space alone does not guarantee that interaction happens but it does make it more accessible. Thus, multidisciplinary teaching in the XJTLU Joint Delivery modules tries to incorporate a variety of methods to allow for interaction, for example, small group discussions and reporting, virtual chat (live, onscreen), concept mapping, role plays, debates, and poster displays to name a few. These types of activities are much more manageable in a flat learning space where the furniture can be manipulated to suit the activity. Student feedback has shown that the use of these "new" flat learning spaces for large groups are popular, and based on observation, it has influenced the teaching style of lecturers to some degree. Additionally, these spaces are in heavy demand and are fully booked during teaching weeks.

For the module LAN004 'Imagining the City' and 'Critical Thinking: The Self and the World' it is and was interesting to contrast the differences and similarities between the learning spaces. Due to the large seminar grouping for the first module, tutors had to facilitate learning in a space that held on average over 100 students. The space also had four distinct pillars in what was a large flat space, necessitating the tutors to move around with a microphone in hand to better ensure that their message could be adequately conveyed and avoid scenarios in which students might feel neglected or alternatively choose seating placements in order to effectively hide. The room also required the use of three projector screens to better ensure that any material visually conveyed could be adequately viewed by all in attendance. In contrast, the learning space for the second module was smaller. With a class size more akin to a regular class normal teaching provisions could be worked around and coteaching was not necessitated so a single tutor could deliver the sessions. 


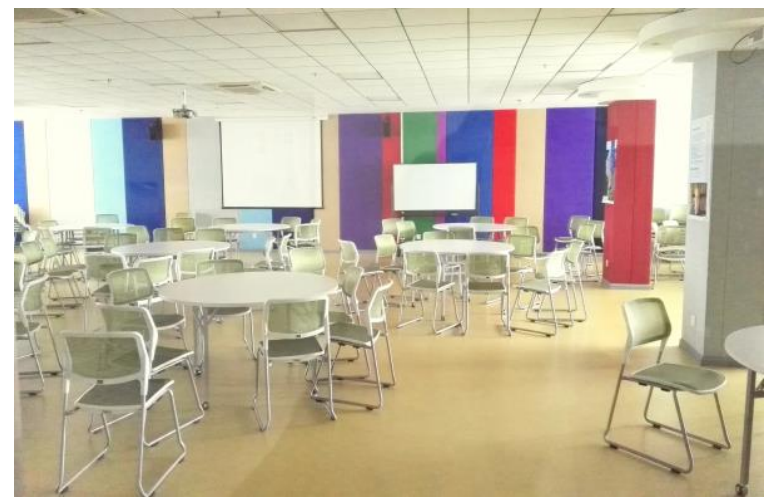

Figure 1. Flat learning space classroom at XJTLU. Capacity 150 students (2016)

\section{Consideration 4: Technology and Techniques}

Taking again, as an example, the Joint Delivery module LAN004, 'Imaging the City', which has been taught in the 2015-2016 academic year at XJTLU, in terms of teaching practice, technology and techniques implemented this course's goal is the integration and further enhancement of $\mathrm{Y} 1$ students communication capabilities and English language skills with more focused elements drawn from Urban Planning and Design cognitive background. As explained in the module specification of LAN004, the aim of this module, which is run by the Language Centre of $\mathrm{XJTLU}$, is to introduce first year students to a range of themes and topics related to the concept of 'The City'. More specifically, the intention is to help students to understand some of the issues which can affect the cities they live in today, and to think about the future development of urban societies. The module also seeks to promote interdisciplinary thinking by making connections between themes as topics are introduced and delivered by different lecturers from both the UPD - Department of Urban Planning and Design and ECC - English, Culture and Communication. A typical teaching week consists of 2 separated sessions: a 2 hours lecture-based session and 2 hours tutorial and practice session, during which the students are exposed to a broad variety of topics and educational tools based on different technologies, and learning and teaching technique and strategies. A commonly adopted teaching tool used in the delivery of the theory-based lectures is PPT based slides, which are still an extremely versatile and useful instrument for both the lecturer and the student. This teaching tool is enhanced and made more effective when it is integrated with the use of video presentations, which are often presented during the class. Collective intensive practical sessions (e.g. free-hand drawing used in the delivery of lectures for LAN004) and group-based discussions are particularly effective in combination with videos and PPT based lectures, as they prove to be especially helpful in clarifying and better explaining some difficult concepts and notions illustrated during the various lectures, also considering the heterogeneity of the background of the students.

For LAN004 this combination of techniques and methods proved to be particularly useful as the topics covered in the lectures illustrated the most relevant key subjects of the discipline of the planning and design of cities as seen by planners and urban designers, by introducing notions related to the changing natures of the city, the various techniques of urban representation (visual and analytical), to proceed to a more detailed outline of the physical and functional elements of the city as a built environment and as an integrated system of complex social and cultural spaces in perpetual change and interaction. This mix also offers greater session diversity and variety, which respond to the need for educators to help students to adapt to a very complex world by teaching them knowledge, but also to help them acquire knowledge by themselves [13]. The implementation of "Active Learning" strategies are integrated with "Peer-to-Peer Learning" strategies, in combination with periodical reviews and frequent general collective feedbacks from the lecturers, so that it is possible to provide a more efficient and rich learning experience while giving students useful suggestions and comments on their performance and achievements at short periods. In this context pre and post lecture tasks, activity completion tracking and reflective podcasts that all of the cohort could access were other initiatives that were undertaken to promote learning. The implementation of active learning strategies is achieved by making materials and additional resources available to the students before/after the lecture (all teaching materials and readings presented for the class and indispensable to help students prepare for the their research are available online on the university web-site platform ICE); in addition the peer-to-peer learning strategies, where all the students can effectively learn through collaborative activities, contribute overall to more efficient and rich learning experience, where the teachers operate as a fundamental catalysts in order to promote and foster interdisciplinary and multidisciplinary learning through creative thinking, effective communication strategies and a balanced collaborative approach.

It is also worth mentioning that among the challenges for the instructors in teaching this module is also the search for a balance and integration of a purely academic learning knowledge, as introduced for instance by the delivery of information and data 


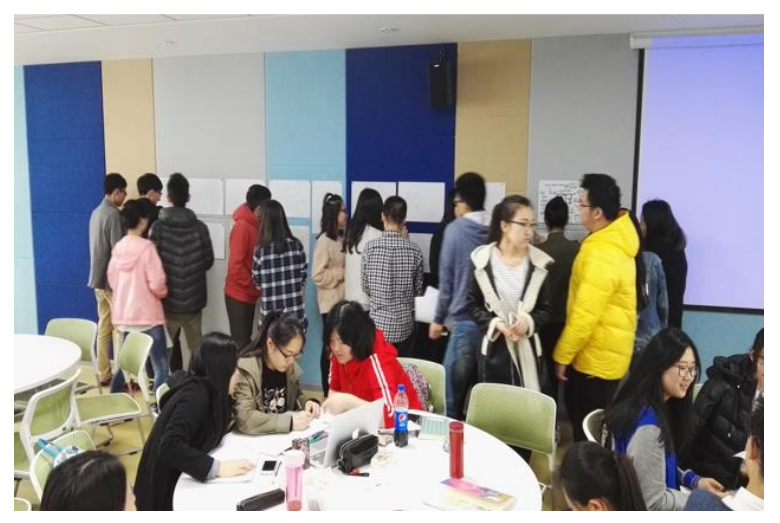

Figure 2. Flexible learning spaces that can accommodate large class sizes at XJTLU to fit different teaching tasks (2016)

provided through the lectures, the readings, and other sources delivered in class, with a more practical learning experience needed by the students. Students, especially in the field of urban planning and design, educated and trained to test solutions and to conceptualize and translate subjective ideas and abstract notions into more concrete forms of knowledge during the university activities, be them in studios, workshops or seminars, eventually need to test their opinions and beliefs and confront them with the reality of world outside the classroom, in all its complexity. For instance, urban planning and design, traditionally as a means of government intervention and discipline which aims at shaping the forms of the cities, has been endowed with the justification to provide the forms and guidance for development as serving a common good or public/collective interest. However, governance nowadays increasingly takes place in a polycentric manner when the state/government plays a much less dominant role than before [14]. It provides the essential institutional framework within which decisions are made through complex interplay of different stakeholders and actors, many of whom are non-state agencies and individuals [15]. Therefore, it appears evident that no single actor is legitimate enough to direct the change of the society due to the complicated relationships among multiple actors and policy-making processes given that legitimacy has to be socially constructed, not being granted by any state actions. That means that relations, networks, process and participation all become the key words that planners and simple citizens need to grasp in order to understand the scale of challenges that we face in a more and more urbanized and complex world, a reality with which the students will have sooner or later to deal with, both as professionals or/and as effective and responsible citizens.

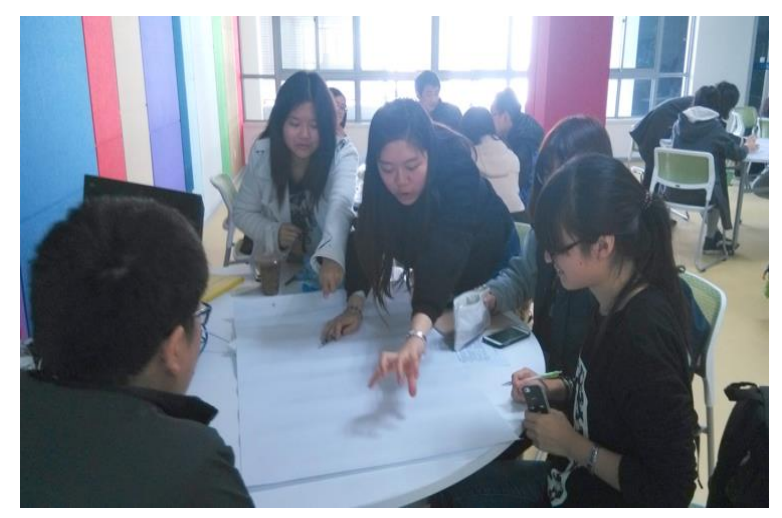

Figure 3. Collaborative activities in the same classroom at XJTLU. Capacity is 150 (2016)

\section{Conclusion}

This paper has briefly highlighted the rationale behind the establishment of Joint Delivery modules at XJTLU and considered some of the curriculum and assessment considerations that ought to be considered on them, and the evolving pedagogic practices which are employed by the practitioners who teach them. The paper has also provided a variety of collaborative teaching practice insights and also a number of ideas for how to better improve on what is currently offered in an exciting evolving, innovative and potentially influential area of learning and teaching. To do so, consideration of how to use different learning and teaching spaces, how to integrate at times diverse topical elements, how and when to incorporate technology, and also how to work with people from very different specialist and educational backgrounds was provided. At the same time the reflective thoughts of those who have taught on these modules were offered in terms of what was deemed to work well and what could be improved, and also in terms of how some of the potential challenges facing multidisciplinary teaching and learning collaborative practice might be either avoided or overcome in the years ahead.

\section{References}

[1] Antonietti, A., Confalonieri, E., and Marchetti, A. (Eds.). (2014). Reflective Thinking in Educational Settings: A Cultural Framework. Cambridge University Press.

[2] Astin, A. W. and Antonio, A. L., (2012). Assessment for excellence: The philosophy and practice of assessment and evaluation in higher education. Rowman \& Littlefield Publishers. 
[3] Brookfield, S. D., and Preskill, S., (2012). Discussion as a way of teaching: Tools and techniques for democratic classrooms. John Wiley \& Sons.

[4] Brown, J. (1995) The Elements of Language Curriculum. A Systematic Approach to Program Development. Boston: Heinle and Heinle.

[5] Darder, A., Baltodano, M., and Torres, R. D. (Eds.). (2003). The critical pedagogy reader. Psychology Press.

[6] Freire, P., and Macedo, D., (2005). Literacy: Reading the word and the world. Routledge.

[7] Giroux, H. A., (2011). On critical pedagogy. Bloomsbury Publishing USA.

[8] Jordan, E., (2016) Departmental/LC joint delivery module options for 2016-17. Internal XJTLU report. Unpublished.

[9] Levinson, M., (2012). No citizen left behind (Vol. 13). Harvard University Press.

[10] Moll, L. C., Amanti, C., Neff, D., and Gonzalez, N., (1992). Funds of knowledge for teaching: Using a qualitative approach to connect homes and classrooms. Theory into practice, 31(2), 132-141.

[11] Richards, J., (1995). Easier Said than Done: An Insiders Account of a Textbook Project. In Hidalgo, A. Hall, D. Jacobs, G. (Eds.). Getting Started: Materials Writers on Materials Writing (95-135). Singapore: SEAMEO RELC.

[12] Rust, C., (2006). Re-designing Universities: Social Learning Space: Introduction to Symposium. Available from: http://www2.warwick.ac.uk/fac/cross_fac/iatl/cetl/n ews/symposium (Access Date: 6 April, 2016).

[13] Norman J. J., (2011), Learning for a Complex World: A Lifewide Concept of Learning, Education and Personal Development. Author House Publishing

[14] Report UN-Habitat, (2001). Cities in A Globalizing World - Global Report on Human Settlements 2001. Available from https://unhabitat.org/year/2001/ (Access Date: 1 September, 2017).

[15] Wilson, D.J. and Game, C., (2011). Local Government in the United Kingdom. Basingstoke: Palgrave Macmillan. 